Growth regimes of porous gold thin films deposited by magnetron sputtering at oblique incidence: from compact to columnar microstructures

This article has been downloaded from IOPscience. Please scroll down to see the full text article.

2013 Nanotechnology 24045604

(http://iopscience.iop.org/0957-4484/24/4/045604)

View the table of contents for this issue, or go to the journal homepage for more

Download details:

IP Address: 150.214.182.8

The article was downloaded on 08/01/2013 at 20:14

Please note that terms and conditions apply. 


\title{
Growth regimes of porous gold thin films deposited by magnetron sputtering at oblique incidence: from compact to columnar microstructures
}

\author{
R Alvarez ${ }^{1}$, J M García-Martín ${ }^{2}$, M Macías-Montero ${ }^{1}$, \\ L Gonzalez-Garcia ${ }^{1}$, J C González ${ }^{1}$, V Rico ${ }^{1}$, J Perlich ${ }^{3}$, J Cotrino ${ }^{1,4}$, \\ A R González-Elipe ${ }^{1}$ and A Palmero ${ }^{1}$ \\ ${ }^{1}$ Instituto de Ciencia de Materiales de Sevilla (CSIC-US), Americo Vespucio 49, E-41092 Seville, Spain \\ 2 IMM- Instituto de Microelectrónica de Madrid (CNM-CSIC), Isaac Newton 8, E-28760, Tres Cantos, \\ Madrid, Spain \\ ${ }^{3}$ HASYLAB at DESY, Notkestraße 85, D-22603 Hamburg, Germany \\ ${ }^{4}$ Departamento de Física Atómica, Molecular y Nuclear, Universidad de Sevilla, \\ Avenida Reina Mercedes s/n, E-41071 Seville, Spain \\ E-mail: rafael.alvarez@icmse.csic.es
}

Received 24 September 2012, in final form 3 December 2012

Published 8 January 2013

Online at stacks.iop.org/Nano/24/045604

\begin{abstract}
Growth regimes of gold thin films deposited by magnetron sputtering at oblique angles and low temperatures are studied from both theoretical and experimental points of view. Thin films were deposited in a broad range of experimental conditions by varying the substrate tilt angle and background pressure, and were analyzed by field emission scanning electron microscopy and grazing-incidence small-angle $\mathrm{x}$-ray scattering techniques. Results indicate that the morphological features of the films strongly depend on the experimental conditions, but can be categorized within four generic microstructures, each of them defined by a different bulk geometrical pattern, pore percolation depth and connectivity. With the help of a growth model, a microstructure phase diagram has been constructed where the main features of the films are depicted as a function of experimentally controllable quantities, finding a good agreement with the experimental results in all the studied cases.
\end{abstract}

S. Online supplementary data available from stacks.iop.org/Nano/24/045604/mmedia

(Some figures may appear in colour only in the online journal)

\section{Introduction}

It is well known that gold has endothermic oxygen chemisorption energy, thus being inert towards oxidation reactions [1]. In this aspect, nanoporous gold has attracted much attention in science and technology for its functionality as a catalyst for carbon monoxide oxidation. In [2], for instance, it is found that the presence of a high density of geometric steps and kinks in nanoporous gold is essential to activate chemical reactions with the advantage of a high thermal stability against coarsening, thus presenting advantages over the catalytic activity of gold nanoparticles. Consequently, the synthesis of gold thin films with large surface area and with valleys that penetrate deep into the material seems relevant in order to enhance these properties. Although nanoporous gold has usually been synthesized by the chemical dealloying of $\mathrm{Au}_{35} \mathrm{Ag}_{65}$ (see for instance [2, 3]), the possibility to use plasma-assisted deposition techniques would be desirable, not only because it might allow a better control on the surface and pore percolation features, but also 
from environmental and industrial points of view. This paper is aimed at theoretically and experimentally studying the growth of nanoporous gold thin films by magnetron sputtering at low temperatures, and at analyzing the percolation depth and connectivity of the surface valleys that penetrate into the film for enhanced catalytic applications.

In [4] we theoretically and experimentally studied the growth of gold thin films deposited by magnetron sputtering at oblique angles and low temperatures by varying the background plasma pressure. There, we found an intrinsic connection between film microstructure and the degree of thermalization of sputtered particles by interaction with gas and plasma heavy particles, described by the quantity $\Xi$ and defined as

$$
\Xi=\frac{L}{v \lambda}
$$

where $L$ is the target-film distance, $\lambda$ the mean free path of sputtered particles, and $v$ the number of elastic collisions required to thermalize a sputtered atom. In this way, when $\Xi \ll 1$, the sputtered particles do not experience many collisions and mainly keep their original directionality and energy just after their sputtering from the target. Under these conditions, films with a tilted columnar microstructure, very similar to those obtained by electron beam assisted evaporation at oblique angles [5-7], were grown. When $\Xi \gg 1$, on the other hand, the sputtered particles experience enough collisions to become thermalized and arrive at the film surface with an isotropic velocity distribution function. These working conditions yielded porous films with vertical column-like structures, similar to those obtained for $\mathrm{TiO}_{2}$ deposited at the back side of the substrate holder in [8].

The gold microstructures obtained in [4] contrast with typical microstructures found at normal angle depositions, where films are usually compact and homogeneous [8]. Due to the variety of microstructures found by magnetron sputtering at low temperatures, in this paper we broaden the scope of previous works $[4,8]$ and systematically analyze the thin films deposited by magnetron sputtering at oblique angles for a wide range of values of the substrate tilt angle, $\sigma$, and background pressure, $p_{\mathrm{g}}$. Our aim is therefore a complete experimental and theoretical classification of the possible growth modes of gold thin films and the study of the microstructure of the films, including the porous structure, percolation depth and connectivity for catalytic applications. As a result, we have found that the whole set of experimental gold thin film microstructures can be described through only four generic microstructures, based on which we have constructed a phase diagram where the morphological features of the films are connected with the values of $\sigma$ and $\Xi$. Particular attention has been paid to the development of geometrical patterns in the bulk of the films as well as to the size and percolation depth of surface valleys, an aspect that has received little attention in previous studies in the literature for plasma-assisted depositions of thin films [7, 9-11].

We believe that the research presented in this paper possesses relevance not only for the fundamental knowledge regarding the microstructure formation and growth of a)

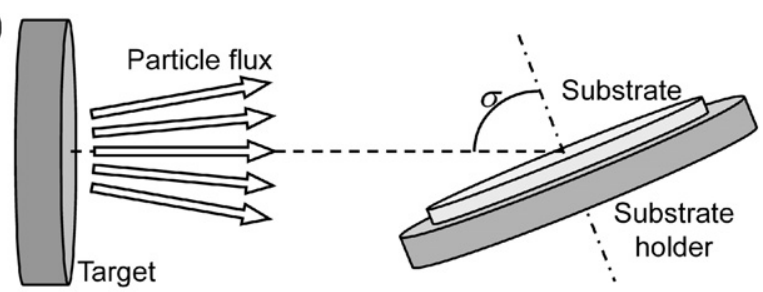

b)

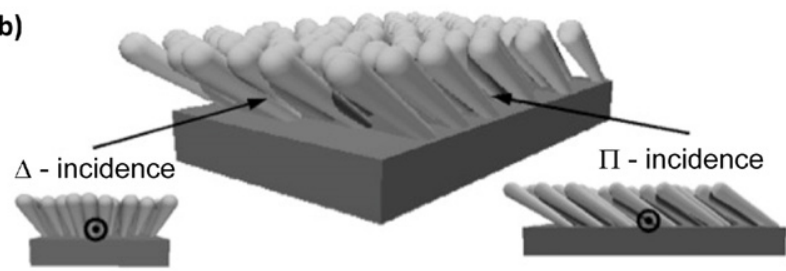

Figure 1. (a) Experimental setup; (b) definition of the $\Delta$ and $\Pi$ directions on the films.

magnetron sputtered thin films at low temperatures and oblique angles, but, as evidenced in previous work dealing with the fabrication of thin films with a localized surface plasmon resonance behavior [12-14], also for its potential use for the fabrication of metal films with special optical properties. We also believe the films we analyze are of the outmost interest for applications concerning the catalytic activity of nanoporous gold and, in particular, for carbon monoxide oxidation applications [2].

\section{Experimental setup}

Gold thin films were deposited by the magnetron sputtering technique using a $3.8 \mathrm{~cm}$ diameter gold target and argon as sputter gas. The base pressure of the chamber is in the mid $10^{-7}$ Pa range. A $2 \mathrm{~cm}^{2}$ ultrasonically cleaned $\operatorname{Si}(100)$ substrate was placed at $L=0.19 \mathrm{~m}$ from the target, and tilted with respect to its normal (see figure 1(a)). The tilt angles of the substrate were set to different values ranging from $\sigma=45^{\circ}$ to $87.5^{\circ}$. The argon gas filled the whole deposition chamber and its pressure was set from 0.15 to $4 \mathrm{~Pa}$, with the power and the deposition time being kept constant at $100 \mathrm{~W}$ and $1800 \mathrm{~s}$ respectively. The visible plasma glow was always many centimeters away from the substrate holder (the glow volume was about 50-75 $\mathrm{mm}$ next to the target) and the film temperature during the sputtering process was always below $350 \mathrm{~K}$.

Films were characterized by field emission scanning electron microscopy (FESEM) as well as by grazingincidence small-angle $x$-ray scattering (GISAXS). Since film microstructure was rapidly altered by the electron beam during measurement when using the former technique, we used short exposure times in order to obtain reliable images. The GISAXS technique was implemented at the synchrotron beamline BW4 of the DORIS III storage ring (DESY, Hamburg) using a wavelength of $0.138 \mathrm{~nm}$ and a sample-to-detector distance of $2.175 \mathrm{~m}$ [15]. A moderate microbeam focusing was achieved using beryllium refractive lenses (beam size $42 \mu \mathrm{m} \times 22 \mu \mathrm{m}$ ). The scattering signal was recorded with a $2 \mathrm{D}$ detector (MAR CCD camera with 
$79.1 \mu \mathrm{m}$ lateral pixel size). For each sample two different patterns were recorded by placing the films oriented according to the $\Delta$ and $\Pi$ configurations, defined in figure $1(\mathrm{~b})$. In the $\Delta$ configuration the beam does not see any possible tilting of the microstructural features, while in the $\Pi$ configuration it impinges perpendicular to the plane containing the tilting angle of the microstructural features.

\section{Growth model}

The Monte Carlo growth model is similar to that employed in [4]. It considers the deposition of gold atoms on a two-dimensional substrate that defines the $x-y$ plane of coordinates, whereas the $z$ axis is defined by the direction perpendicular to it. The three-dimensional space is divided into an $N_{\mathrm{L}} \times N_{\mathrm{L}} \times N_{\mathrm{H}}$ grid, where each cell has the value 1 if it contains a deposited gold atom and 0 otherwise. Each cell, therefore, represents a gold atom in the network. Cell width is estimated by the typical distance between atoms in the material $(0.4 \mathrm{~nm})$.

Each deposition particle is thrown towards the substrate from an initial random position above the film, following the direction defined by the spherical angles $\theta$ and $\varphi$, where $\theta \in$ $[0, \pi / 2)$ is the polar angle of incidence $(\theta=0$ is the direction normal to the substrate) and $\varphi \in[0,2 \pi)$ is the azimuthal angle. The movement of the particle continues along a straight line, assuming periodic boundary conditions, until it hits the surface, where it sticks. The angles $\theta$ and $\varphi$ are randomly calculated for each deposition particle by defining an incident angle distribution function per unit time and unit surface, $I(\Omega)$, with $\mathrm{d} \Omega=\sin \theta \mathrm{d} \theta \mathrm{d} \varphi$ being the differential solid angle. In order to estimate the magnitude $I(\Omega)$ we have employed the SIMTRA code that allows the determination in the deposition system geometry of the angular distribution function of the deposition particles that arrive at the substrate [16]. A Moliére type potential was taken to describe the interaction between $\mathrm{Au}$ and $\mathrm{Ar}$ atoms. Once the function $I(\Omega)$ is calculated, the model is solved for values of $N_{\mathrm{L}}$ and $N_{\mathrm{H}}$ up to 2000 .

Due to the separation between the plasma glow and the substrate holder in all the experimental conditions and the absence of electronegative gases in the reactor, we have not taken into account any plasma ion-surface interaction in the growth model [17, 18]. Furthermore, due to the low temperature of the films during growth, no surface mobility has been considered, as the ratio $T_{\mathrm{s}} / T_{\mathrm{m}} \sim 0.2, T_{\mathrm{s}}$ and $T_{\mathrm{m}}$ being the film temperature during growth and the gold melting temperature, respectively, corresponds to the so-called Zone I in the SZM model, where the deposition is dominated by surface shadowing $[19,20]$. These approximations will be discussed in section 4 .

The simulated microstructures were characterized by taking into account the geometrical patterns appearing in the bulk of the films, and also as a function of the size and percolation depth of the pores in the material. According to the IUPAC [21], we distinguish micropores, with typical entrance sizes smaller than $2 \mathrm{~nm}$, and mesopores, with entrance sizes larger than $2 \mathrm{~nm}$. Moreover, we distinguish pores occluded in the material, as well as those connected with the surface.

\section{Results and discussion}

\subsection{Theoretical results}

In our analysis, rather than the experimentally controllable quantity, $p_{\mathrm{g}}$, we employ the non-dimensional parameter $\Xi$, defined in equation (1), with $L=0.19 \mathrm{~m}$ the distance between the cathode and the film, $v=12$ the number of collisions required to thermalize the gold atom in the argon environment [22], and $\lambda=k_{\mathrm{B}} T_{\mathrm{g}} / p_{\mathrm{g}} \sigma_{\mathrm{Au}-\mathrm{Ar}}$ the mean free path of the gold atom, with $k_{\mathrm{B}}$ the Boltzmann constant, $T_{\mathrm{g}}=600 \mathrm{~K}$ the spatially averaged value of the gas temperature, and $\sigma_{\mathrm{Au}-\mathrm{Ar}}$ the cross-section for an elastic scattering of an $\mathrm{Au}$ atom on an Ar atom $\left(\sigma_{\mathrm{Au}-\mathrm{Ar}}=3.25 \times 10^{-19} \mathrm{~m}^{2}\right)$. Consequently, under our conditions, we estimate $\Xi \sim 0.67 \times p_{\mathrm{g}} \mathrm{Pa}^{-1}$.

Simulated film microstructures possess different features that depend on the values of $\Xi$ and $\sigma$. Figure 2 shows a series of snapshots corresponding to four different calculations obtained for extreme values of these two parameters. These simulations have been selected because they are representative of whole sets of calculated thin films with similar microstructural and pore features. These four generic microstructures depicted in figures 2 (a)-(d) will be designated here as $\alpha, \beta, \gamma$, and $\delta$, and are characterized by the following features:

- $\alpha$-type microstructure. The film is compact without any well defined geometrical pattern in the bulk of the material, and has a very small density of pores (see figure 2(a)).

- $\beta$-type microstructure. The film is fairly compact, but surface valleys percolate from the very surface of the film to near the substrate through vertical tilted mesopores (see figure 2(b)). This structure could also be seen as a tilted highly coalescent columnar structure (i.e., columns are not isolated from each other but are always touching), whereas mesopores can be devised as the empty space between the coalescent columns.

- $\gamma$-type microstructure. The film possesses similarities with the $\beta$-type microstructure, but now surface valleys and mesopores are larger and the columns appear well separated and isolated from each other or form small groups or islands (see figure 2(c)).

- $\delta$-type microstructure. This film is characterized by vertical coalescent column-like structures, with a high density of micro- and mesopores occluded in the material, and with cavern-like surface patterns elongated in the vertical direction that penetrate deep into the bulk (see figure 2(d)).

Simulations of the growth of these microstructures are found in the form of video files as supplementary material (available at stacks.iop.org/Nano/24/045604/mmedia). In order to further understand the main features of the above mentioned four generic microstructures, we have studied the connectivity of mesopores in the materials, i.e., we have determined whether a particular void within the material is occluded (completely surrounded by material), or there is a continuum path that connects the void with the film surface (air) through mesopores. In figure 3(a) we have depicted the 

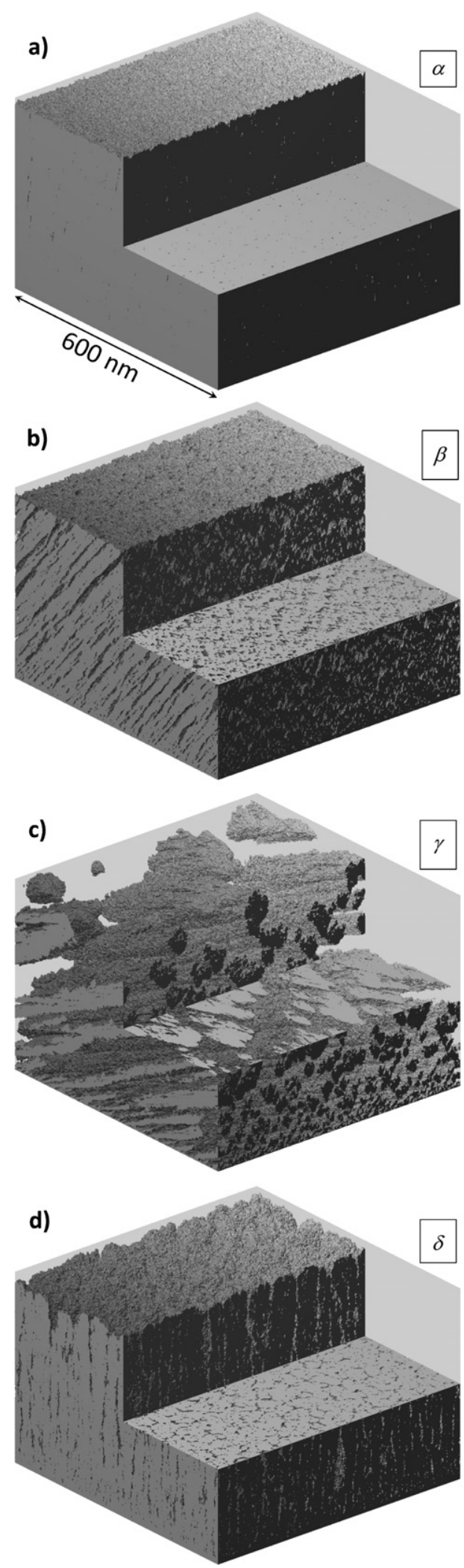

Figure 2. Typical microstructures found in the simulations. (a) $\alpha$ type (simulation conditions $\Xi=0.1$ and $\sigma=45^{\circ}$ ), (b) $\beta$ type (simulation conditions $\Xi=0.1$ and $\sigma=70^{\circ}$ ), (c) $\gamma$ type (simulation conditions $\Xi=0.1$ and $\sigma=89^{\circ}$ ), (d) $\delta$ type (simulation conditions $\Xi=10$ and $\sigma=0^{\circ}$ ).

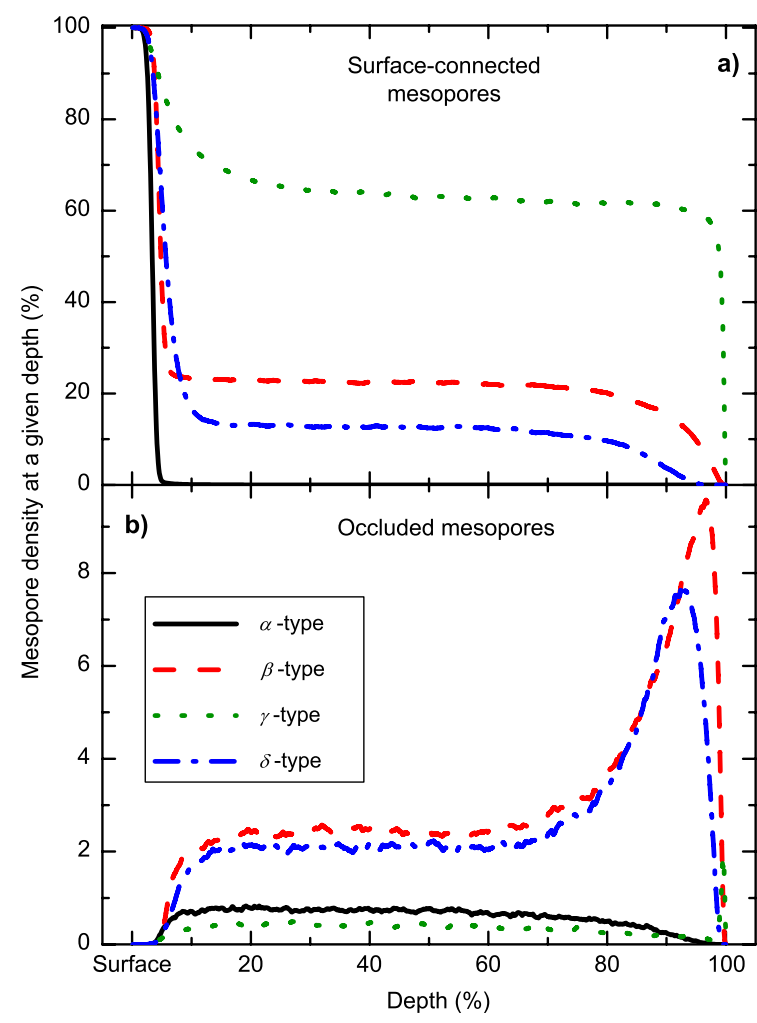

Figure 3. Local density of mesopores at a given depth for the generic microstructures in figure 2, as a function of depth.

(a) Surface-connected mesopores; (b) occluded mesopores.

local density of surface-connected mesopores at a given depth in each of the four characteristic microstructures, calculated as follows: (i) once the three-dimensional map for each film was obtained, we calculate the size and connectivity of the pores and only consider those large enough to be mesopores, (ii) we do not take into account those mesopores that are occluded, i.e., those not connected with the film surface (air), and (iii) for each slice of material at a given depth we calculate the volume of surface-connected mesopores in comparison with the total volume of the slice. At the film surface all of them show a high surface-connected volume, which corresponds to the surface roughness region, always present in the films. After this region, the surface-connected volume of the $\alpha$-type microstructure rapidly drops to zero, indicating that pores in the bulk of the film are always occluded. Conversely, the $\beta$-type microstructure shows a surface-connected mesopore volume of about $25 \%$ after the roughness region, which remains constant up to depths near the substrate. This is more prominent in the $\gamma$-type microstructure where it reaches about $65 \%$ in the bulk of the film, a value which is reasonable due to the existence of isolated columnar structures. Finally, the $\delta$-type microstructure shows the existence of surface-connected mesopores within the material, which now amount to about $10 \%$ in the bulk of the film, i.e. a much lower accessibility to bulk features from the surface. For illustration purposes, we present the local density of occluded mesopores as a function of depth for each abovementioned characteristic microstructure in figure 3(b). In general, their density is quite low, most of the pores 


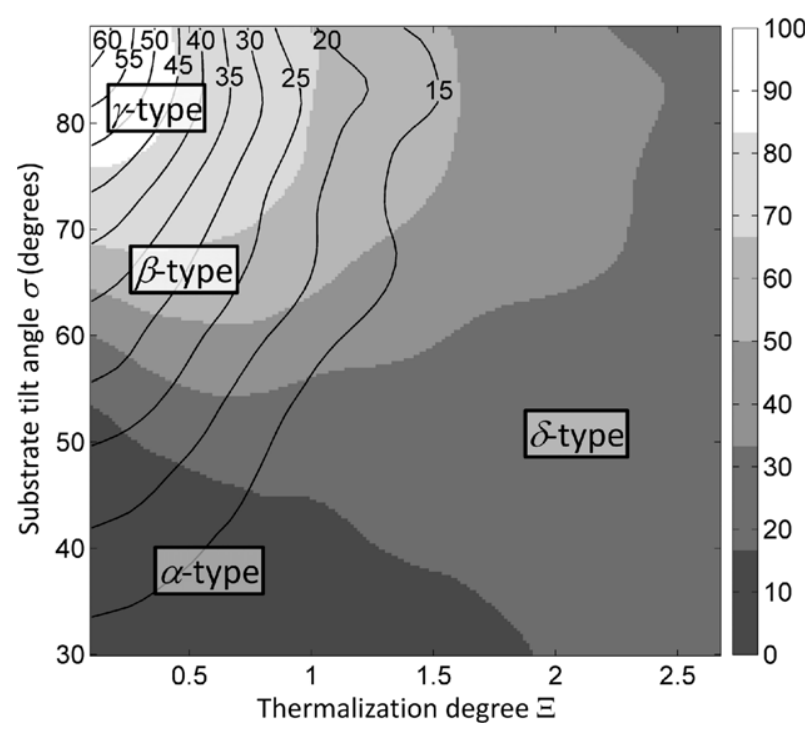

Figure 4. Microstructuring regime phase map predicted by the growth model as a function of $\Xi$ and $\sigma$. The colors indicate the average penetration depth of the surface valleys into the film through mesopores (\%), whereas lines denote the tilt angle of the structures (columns or mesopores) in the bulk of the film (degrees). Labels ' $\alpha$-type', ' $\beta$-type', ' $\gamma$-type and ' $\delta$-type' correspond to the regions where the generic microstructures depicted in figure 2 are found.

being surface connected. This is evident for the $\alpha$ - and $\gamma$-type microstructures, with a local density always below $1 \%$, whereas the $\beta$ - and $\delta$-type microstructures present a density around $2 \%$ in the bulk that increases near the substrate to values below $10 \%$.

Once the four generic microstructures generated by the model have been characterized, we systematically run simulations in multiple conditions in order to calculate the different microstructures as a function of $\Xi$ and $\sigma$. Results are depicted in figure 4, where we present the obtained results in the form of calculated microstructure phase diagram. Thus, when $\Xi<1$ and for the highest values of $\sigma$ we find that the highly directional deposition particle flow develops surface mounds that serve as seeds for columnar structures. These columns grow tilted from about $45^{\circ}$ to about $60^{\circ}$ with respect to the normal, the average percolation depth of surface valleys being above $80 \%$ of the total film thickness, resembling $\gamma$-type microstructures. For lower values of $\sigma$, and maintaining $\Xi<1$, surface mounds also appear at the initial stages of growth. They serve as seeds, although the lower value of the substrate tilt angle causes columns to grow closer and to merge, leaving many voids within the material, some of them connected with the film surface. These voids are tilted with respect to the normal and have an average penetration depth of about 50-70\%, resembling a typical $\beta$-type microstructure. For $\Xi<1$ and even lower values of $\sigma$, the growth is characterized by the lack of formation of large surface grains in the first stages of growth, resembling a typical $\alpha$-type compact microstructure, with a penetration depth of surface features lower than $20 \%$. Interestingly, some occluded pores may grow with a tilt angle that depends on the actual values of $\Xi$ and $\sigma$, and this is why there are solid lines in this region of figure 4. As $\Xi$ increases, the deposition particles arrive at the film surface with higher isotropy, until the resulting microstructure becomes independent of $\sigma$, which corresponds to the region of formation of the $\delta$-type microstructure. This region is characterized by the formation of surface grains that grow vertically and that coalesce from the very early stages of growth. In the extreme cases with $\Xi \gg$ 1, a microstructure similar to that in figure 2(d) is obtained, with an average penetration depth of surface features around $20 \%$. We have to underline here that all the simulations performed for figure 4 were done on films with thickness up to $400 \mathrm{~nm}$, and that no interdependence between microstructure and film thickness has been found.

The microstructure phase diagram presented in figure 4 depicts the main morphological features of the microstructures of films prepared by magnetron sputtering as a function of $\Xi$ and $\sigma$, at low temperatures. Next, we present a thorough experimental study of multiple gold thin films deposited as described in section 2 that will be employed to assess its accuracy.

\subsection{Comparison with experimental data}

We split the analysis of the films into three sets according to the value of $\Xi$ during the deposition. The first set corresponds to films deposited under the conditions $p_{\mathrm{g}}=$ $0.15 \mathrm{~Pa}(\Xi \sim 0.1)$, and $\sigma=45^{\circ}, 60^{\circ}, 70^{\circ}, 80^{\circ}, 85^{\circ}$ and $87.5^{\circ}$. According to figure 4 , the simulations indicate that by increasing the value of $\sigma$ a transition from an $\alpha$-type to a $\beta$-type microstructure is obtained when $\sigma \sim 60^{\circ}$. Similarly, a transition from a $\beta$-type to a $\gamma$-type microstructure occurs when $\sigma \sim 80^{\circ}$. In figures 5(a)-(f) we show the cross-sectional FESEM images of these films along the $\Pi$ direction (see the scheme in figure 1(b)). Figure 5(a) corresponds to a situation with $\sigma=45^{\circ}$, where we find a compact film with no apparent geometrical patterns in the bulk. For the case with $\sigma=60^{\circ}$, figure $5(\mathrm{~b})$ shows a structure similar to that in figure 5(a), although now some tilted grooves appear in the microstructure, which become more evident in figure 5(c), where $\sigma=70^{\circ}$. In this latter case, although the structure seems compact, a coalescent column-like microstructural arrangement is depicted ranging from the very surface of the film to near the substrate. In agreement with figure 4 , these experimental results confirm a transition between the $\alpha$-type and $\beta$-type microstructures for $\sigma \sim 60^{\circ}$.

In figure 5(d) we depict the FESEM image of the film deposited at $\sigma=80^{\circ}$. In contrast with the case where $\sigma=$ $70^{\circ}$ (see figure 5(c)), we find a clearly tilted columnar microstructure with columns that become progressively isolated and better defined in the films deposited at $\sigma=$ $85^{\circ}$ and $\sigma=87.5^{\circ}$ (figures 5(e) and (f), respectively). This tendency is more evident in figures 6(a)-(d), where we show the FESEM images of the films viewed from the $\Delta$ direction for $\Xi \sim 0.1$ and $\sigma=70^{\circ}, 80^{\circ}, 85^{\circ}$ and $87.5^{\circ}$, respectively. The film microstructure depicted in figure $6(\mathrm{a})\left(\sigma=70^{\circ}\right)$ indicates the existence of well packed coalescent columns, that become gradually isolated when $\sigma$ increases (figures 6(b)-(d)). Therefore, in agreement with 

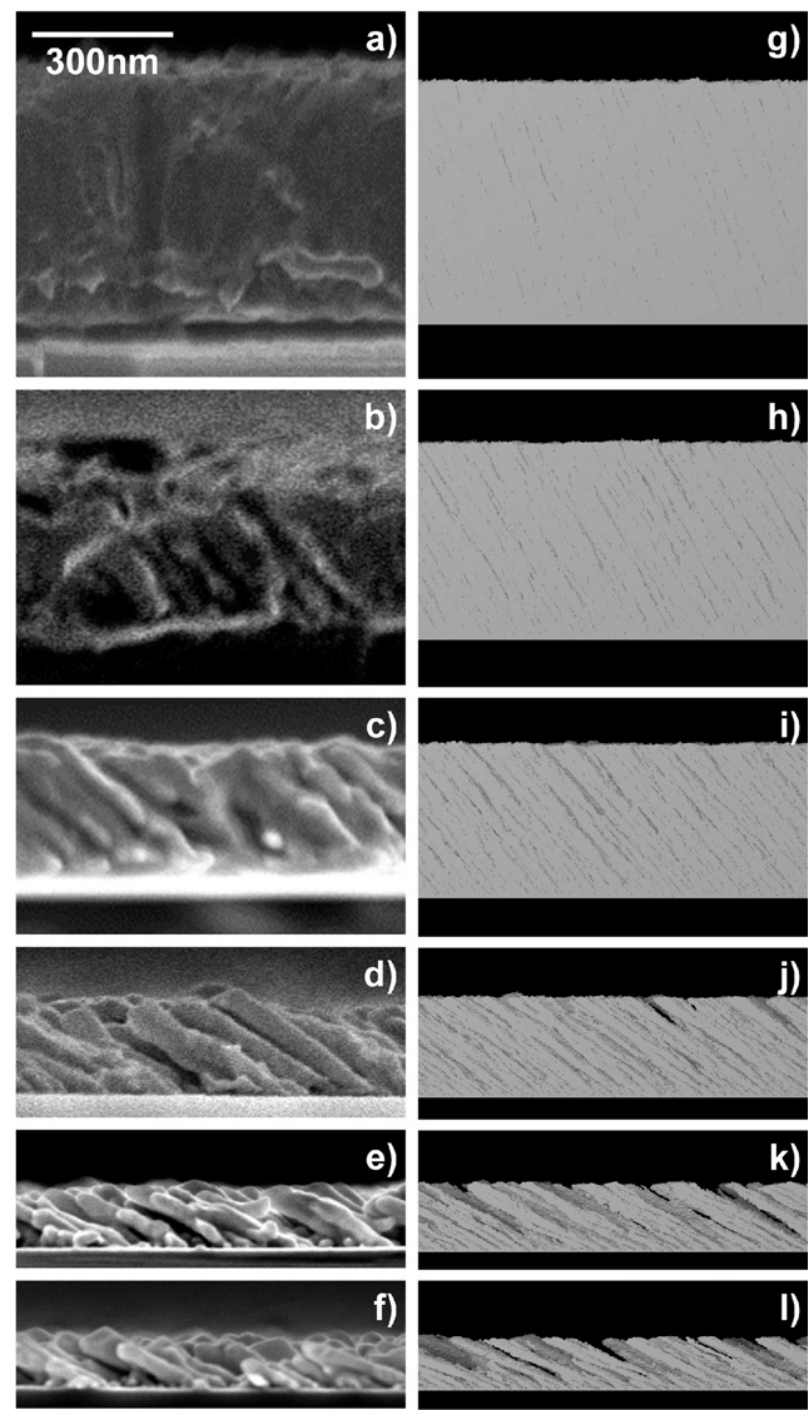

Figure 5. FESEM cross-section images, along the $\Pi$ direction, of films deposited in the conditions $p_{\mathrm{g}}=0.15 \mathrm{~Pa}(\Xi=0.1)$ and (a) $\sigma=45^{\circ}$, (b) $\sigma=60^{\circ}$, (c) $\sigma=70^{\circ}$, (d) $\sigma=80^{\circ}$, (e) $\sigma=85^{\circ}$ and (f) $\sigma=87.5^{\circ}$; (g)-(l) result of the simulations in the same conditions as in (a)-(f), respectively.
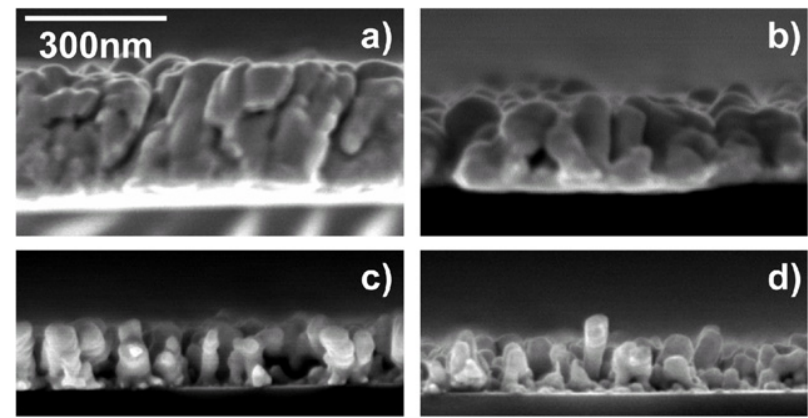

Figure 6. FESEM cross-section images, along the $\Delta$ direction, of films deposited in the conditions $p_{\mathrm{g}}=0.15 \mathrm{~Pa}(\Xi=0.1)$ and (a) $\sigma=70^{\circ}$, (b) $\sigma=80^{\circ}$, (c) $\sigma=85^{\circ}$ and (d) $\sigma=87.5^{\circ}$.

the simulations, we have found a transition from the $\beta$-type to the $\gamma$-type microstructure for $\sigma \sim 80^{\circ}$. This transition is also evidenced by performing GISAXS measurements
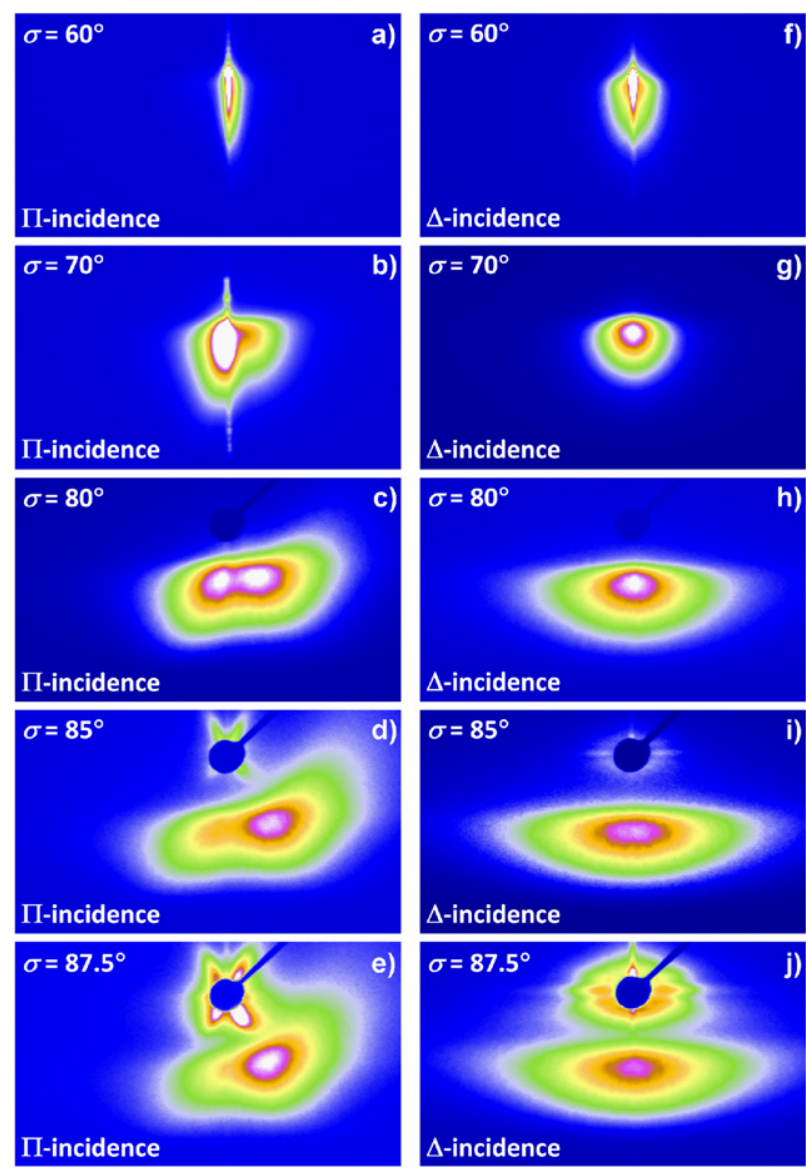

Figure 7. GISAXS spectra of the films for $\Delta$ and $\Pi$ incidence.

on the films: in [23] we have shown that this technique provides information on the microstructural features of tilted columnar thin films and, in some cases, on the existence of critical correlation lengths for oxide thin films prepared by e-beam evaporation. In these previous investigations we showed that an asymmetrical shape of the scattering profile was an indication of a tilted, and therefore asymmetric, arrangement of the film nanocolumns, and that the average value of these correlation distances could be deduced from the distance between the scattering maximum in the largest pattern wing and the center. In the present work, we have essayed a similar analysis for films grown under the conditions $\sigma=60^{\circ}, 70^{\circ}, 80^{\circ}, 85^{\circ}$ and $87.5^{\circ}$ and $p_{\mathrm{g}}=0.15 \mathrm{~Pa}$, ( $\Xi \sim 0.1$ ). The corresponding patterns, gathered in figures 7(a)-(j) have been recorded with the sample oriented in the $\Delta$ and $\Pi$ configurations as explained in section 2 . In the $\Delta$ configuration none of the films present an asymmetric GISAXS pattern, and no displacement of the maximum can be observed. By contrast, in the $\Pi$ configuration, some of the patterns are clearly asymmetric and depict well defined off-center maxima in the largest wing. A more careful evaluation of the patterns can be done by plotting their intensity along a horizontal straight line plotted at the position of the Yoneda peak [24]. In the corresponding plots, reported in figure 8 , it can be clearly appreciated that all the patterns are asymmetric and that the films prepared at $\sigma>70^{\circ}$ depict a clearly defined maximum that appears more separated 


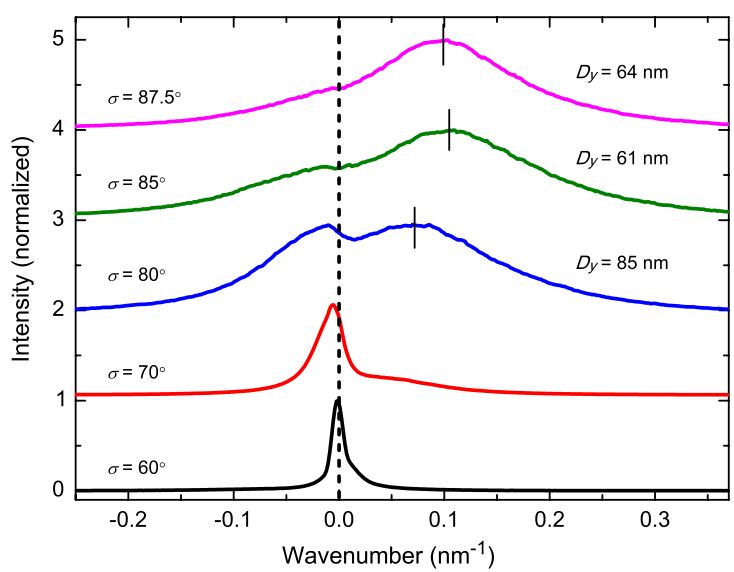

Figure 8. Intensity of the spectra presented in (a)-(e) along a horizontal straight line plotted at the position of the Yoneda peak as a function of the wavenumber, defined as $2 \pi / D$, with $D$ being the wavelength. Maximum values are also shown for films with $\sigma \geq 80^{\circ}$.

from the Yoneda peak as the deposition angle increases Calculations based on the methodology presented in [23, 25 ] permit the calculation of correlation distances of 85,61 and $64 \mathrm{~nm}$ for the films prepared at $80^{\circ}, 85^{\circ}$ and $87.5^{\circ}$, respectively. It must be stressed that the thin films prepared at $70^{\circ}$ and $60^{\circ}$ do not present any well defined maximum apart from the Yoneda peak. This suggests that only for deposition angles greater than $70^{\circ}$ the tilted film microstructure presents well formed individual elements where a clear correlation distance can be determined. We have to stress here that, in general, this distance corresponds to the size of patterns with certain periodicity within the material, and it may refer to either isolated columns or a group of them. Regarding figure 5, the calculated correlation distance is similar to the distance between two consecutive columns in the material. This result corroborates the tendency to form non-coalescent isolated columns in the material for increasing values of $\sigma$, in agreement with the simulations.

In figures $5(\mathrm{~g})-(\mathrm{l})$ we have included the microstructure of the simulated thin films calculated for the same conditions as the experimental cases displayed on the left side (figures 5(a)-(f)). The systematic comparison between experimental and simulated microstructures confirms, in general, a good agreement, although there are slight differences between figures 5(a) and (g). In this case, the model predicts the scattered formation of some tilted occluded pores which do not appear experimentally. This discrepancy points towards the existence of a limited mobility of gold atoms on the film surface making these small occluded voids disappear. Indeed, temperature activated processes or the impingement of plasma ions onto the surface can be responsible for such an effect: our model represents a simple approximation to the actual deposition process, presenting the advantage that no calculated or fitting parameters others than the incident angle distribution function of the deposition particles must be known to run the simulations. In the literature there are numerical codes that consider more complex processes [26, 27], such as thermally activated mechanisms or ion induced

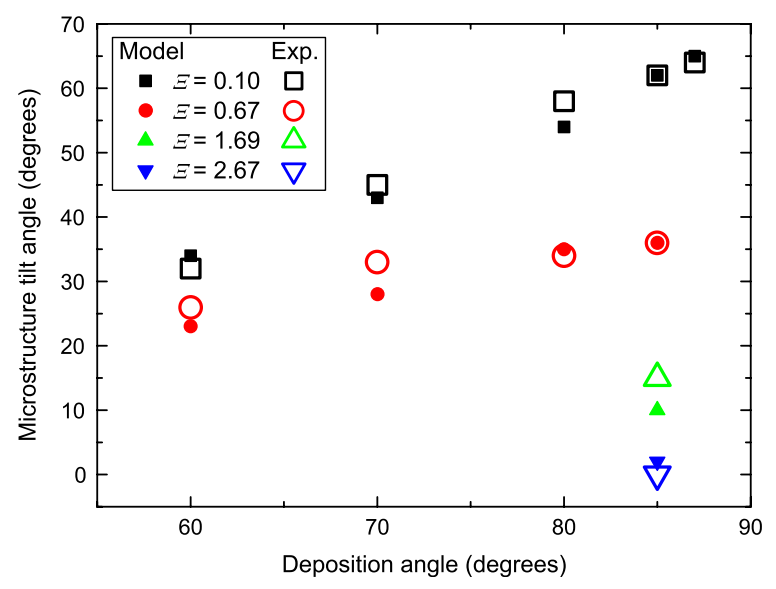

Figure 9. Experimental value of the tilt angle of the studied microstructures as a function of $\sigma$, together with the simulated values.

damage of the films during growth, which require the previous knowledge of activation energies or thresholds for the diverse processes. Although the precise values of these parameters do not appear in the literature in most of the cases, we have solved the NASCAM code in multiple conditions estimating the energy thresholds for these processes through [28] as a test for our model. Results of these simulations corroborate the assumptions of our model and indicate that, in our experimental conditions, surface shadowing determines the main features of the film microstructure, the rest of the processes being responsible for the removal of some occluded voids inside the material. The good agreement between our model and the experimental data is confirmed in figure 9, where we compare the experimental and theoretical values of the tilt angle of the features of the films, always finding a good match between them. Furthermore, the simulations reproduce not only the microstructure evolution but also the decrease in the film thickness when increasing $\sigma$ for equal deposition times (i.e. the same number of sputtered particles in all the cases, a fact that was considered in the simulations).

The second set of studied deposition conditions corresponds to higher values of the deposition pressure. In figures 10(a)-(e) we present the cross-sectional FESEM images of films grown at a pressure of $p_{\mathrm{g}}=1 \mathrm{~Pa}(\Xi \sim$ 0.67 ) and $\sigma=45^{\circ}, 60^{\circ}, 70^{\circ}, 80^{\circ}$ and $85^{\circ}$. For $\Xi \sim 0.67$, figure 4 shows that there is a transition from an $\alpha$-type to a $\beta$-type microstructure when $\sigma \sim 50^{\circ}$. In figure 10(a) we show the FESEM image for $\sigma=45^{\circ}$, where a compact film is depicted. For $\sigma=60^{\circ}$ (figure 10(b)) we find again a compact film, although the bulk possesses now some tilted features that become more isolated for $\sigma=70^{\circ}$, $80^{\circ}$ and $85^{\circ}$ (figures $10(\mathrm{c})-(\mathrm{e})$, respectively). The results of the model for the same deposition conditions are shown in figures 10(f)-(j). They agree well with the experimental images, except for the compact case (figures 10(a) and (f)), where the model still predicts the existence of a few occluded tilted pores that are not visible experimentally. As in the lower pressure case, we believe that this discrepancy is due to the presence of a limited surface mobility, not included 

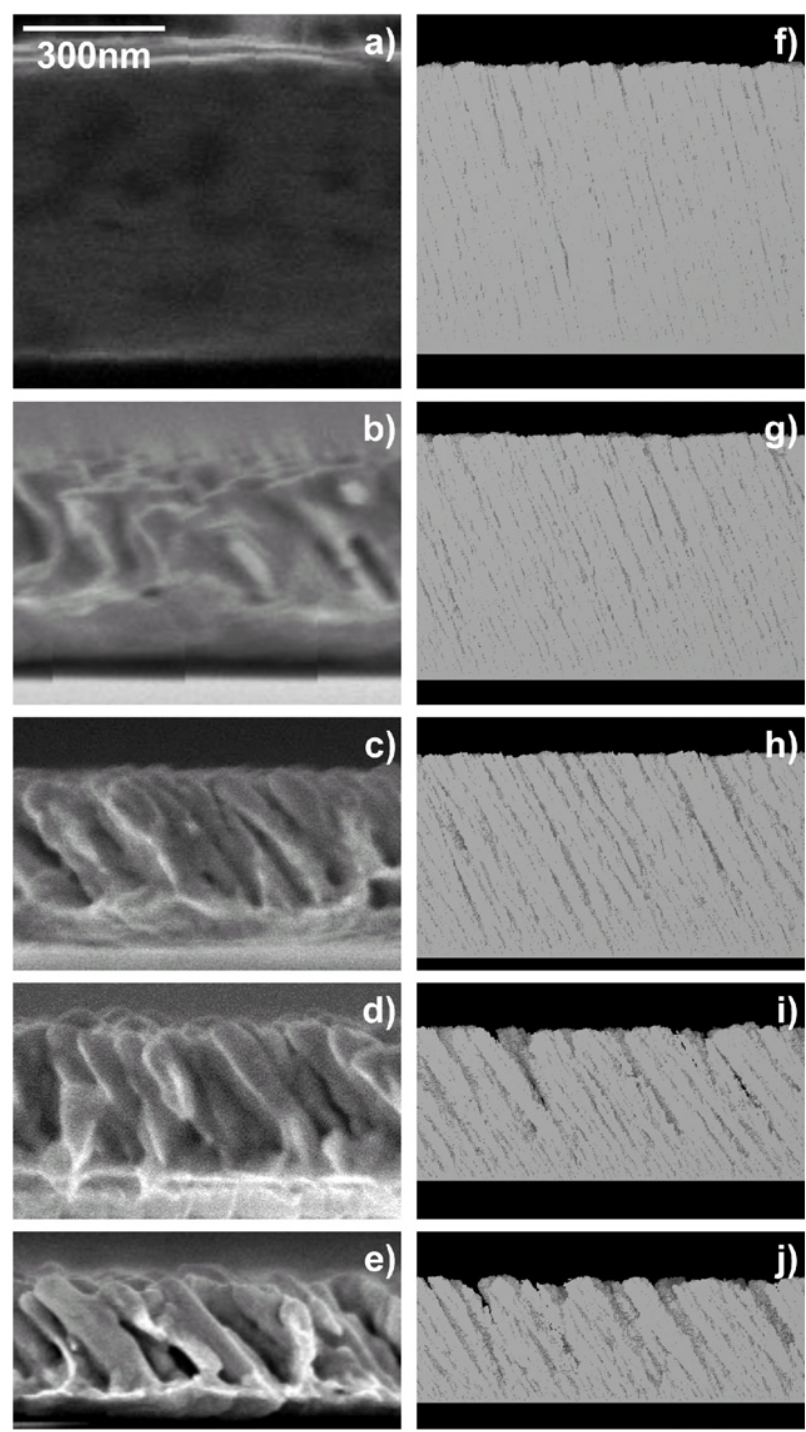

Figure 10. FESEM cross-section images, along the $\Pi$ direction, of films deposited in the conditions $p_{\mathrm{g}}=1 \mathrm{~Pa}(\Xi=0.67)$ and (a) $\sigma=45^{\circ}$, (b) $\sigma=60^{\circ}$, (c) $\sigma=70^{\circ}$, (d) $\sigma=80^{\circ}$ and (e) $\sigma=85^{\circ}$; (f)-(j) results of the theoretical model in the same conditions as in (a)-(e), respectively.

in the model. Except for this discrepancy, the comparison between experimental and simulated values of the tilt angle of the structural features as a function of $\sigma$ is fairly good (see figure 9).

To check the existence of the $\delta$-type microstructure, we have deposited films at higher pressures, $p_{\mathrm{g}}=2.5$ and $4 \mathrm{~Pa}$, for a substrate tilt angle of $\sigma=85^{\circ}$. These conditions result in $\Xi \sim 1.7$ and $\Xi \sim 2.7$, respectively, thus indicating that the deposition flow is highly thermalized by the background gas when arriving at the substrate. The FESEM image of the film for $\Xi \sim 1.7$ appears in figure 11(a), where vertical coalescent structures are apparent. This microstructure becomes more evident in figure 11(b), where we have depicted the FESEM image for the film deposited for $\Xi \sim 2.7$. The results of the simulations under these conditions, shown in figures 11(c) and (d), clearly reproduce the measured microstructure, thus confirming that the experimental cases presented in
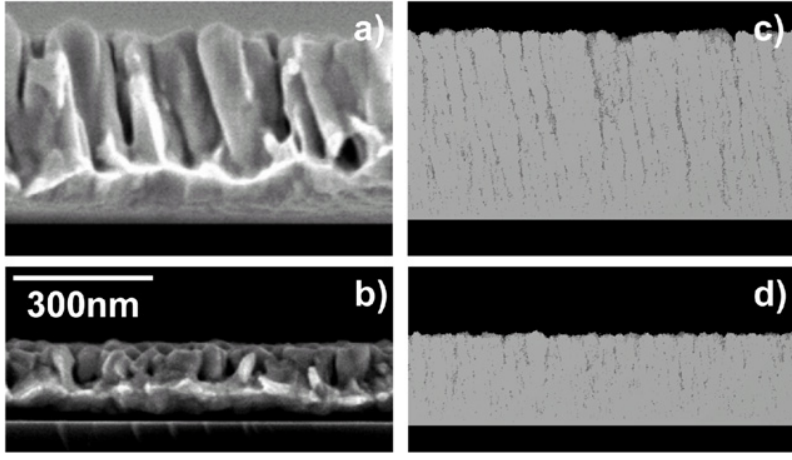

Figure 11. FESEM cross-section images, along the $\Pi$ direction, of films deposited in the conditions (a) $p_{\mathrm{g}}=2.5 \mathrm{~Pa}(\Xi \sim 1.7)$ and $\sigma=85^{\circ}$ and (b) $p_{\mathrm{g}}=4 \mathrm{~Pa}(\Xi \sim 2.7)$ and $\sigma=85^{\circ}$; (c), (d) results of the model in the same conditions as (a), (b), respectively.

figures 11(a) and (b) fit within the predictions of the microstructure phase diagram presented in figure 4. Finally, the comparison between experimental and simulated tilt angles of the patterns appears in figure 9, finding a good agreement. Although the values $\Xi \sim 1.7$ and $\Xi \sim 2.7$ ensure the growth of a $\delta$-type microstructure, the completely vertical columnar microstructure must be understood as a limit case when $\Xi \gg 1$.

As a final remark, we would like to stress that we have studied the different microstructures as a function of $\sigma$ and $\Xi$, when depositions were carried out using an electromagnetic input power of $100 \mathrm{~W}$. In this paper we have not explored the influence of the input power on the film microstructure: this quantity strongly determines the cathode electric potential, and indirectly the sputtering and deposition rates. This means that the influence of mechanisms linked to surface mobility processes controlled by the deposition rate, such as the number of thermally activated atomic jumps on the surface or ions impinging on the surface per deposited particle, would change. In this way, we expect that the higher the deposition rate (i.e. the higher the input power), the lower the influence of surface mobility processes and the less compact the films grow [29]. Nevertheless, as long as that deposition takes place in Zone I of the SZM, these processes should play a minor role, a fact corroborated by the good agreement between our simulations and experimental data.

\section{Conclusions}

In this paper we have studied the different growth regimes of gold thin films deposited by magnetron sputtering by varying the deposition angle and the background pressure as running parameters. For this purpose we have developed a theoretical model that takes into account (i) the elastic scattering of deposition particles on plasma heavy particles in their way from the cathode to the film and (ii) the surface shadowing mechanism. The model predicts that all the film features can be categorized within four generic microstructures, which we have labeled as $\alpha, \beta, \gamma$ and $\delta$ type. Each of them possesses different bulk geometrical pattern, pore connectivity and percolation depth. A microstructure phase diagram has been 
calculated that gives information on the film morphological features as a function of the experimentally controllable parameters. To check the validity of the simulations we have deposited gold thin films under carefully chosen conditions, finding that the microstructures of the synthesized films corroborate the results of the simulations and agree with the microstructure phase diagram. Finally, we would like to stress that we have focused our research on gold thin films, but the aim of this paper is general, and we expect that the results presented in this paper can be generalized to other magnetron sputtered materials.

\section{Acknowledgments}

We thank the Junta de Andalucía (projects P09-CTS- 5189, TEP5283 and P10-FQM-6900), the Comunidad de Madrid (S2009/MAT-1726) and the Ministry of Economy and Competitiveness (projects MAT2011-29194-C02-01, CONSOLIDER CSD2008-00023, MAT2008-06652, MAT201021228, and MAT2010-18447) for financial support.

\section{References}

[1] Hvolbæk B, Janssens T V W, Clausen B S, Falsig H, Christensen C H and Nørskov J K 2007 Nano Today 212

[2] Fujita T et al 2012 Nature Mater. 11775

[3] Fujita T, Qian L, Inoke K, Erlebacher J and Chen M 2008 Appl. Phys. Lett. 92251902

[4] García-Martín J M, Alvarez R, Romero-Gómez P, Cebollada A and Palmero A 2010 Appl. Phys. Lett. 97173103

[5] Alvarez R, Gonzalez-Garcia L, Romero-Gomez P, Rico V, Cotrino J, Gonzalez-Elipe A R and Palmero A 2011 J. Phys. D: Appl. Phys. 44385302

[6] Ye D-X and Lu T-M 2007 Phys. Rev. B 76235402

[7] Asgharizadeh S, Sutton M, Robbie K and Brown T 2009 Phys. Rev. B 79125405

[8] Alvarez R, Romero-Gomez P, Gil-Rostra J, Cotrino J, Yubero F, Palmero A and Gonzalez-Elipe A R 2010 J. Appl. Phys. 108064316

[9] Ferron J, Koropecki R R and Arce R 1987 Phys. Rev. B 357611
[10] Borrás A, Yanguas-Gil A, Barranco A, Cotrino J and González-Elipe A R 2007 Phys. Rev. B 76235303

[11] Warrender J M and Aziz M J 2007 Phys. Rev. B 75085433

[12] Fu J and Zhao Y 2010 Nanotechnology 21175303

[13] Gish D A, Nsiah F, Mc Dermott M T and Brett J 2007 Anal. Chem. 794228

[14] Fu J-X, Collins A and Zhao Y-P 2008 J. Phys. Chem. C 11216784

[15] Roth S V, Döhrmann R, Dommach M, Kuhlmann M, Kröger I, Gehrke R, Walter H, Schroer C, Lengeler B and Müller-Buschbaum P 2006 Rev. Sci. Instrum. 77085106

[16] Van Aeken K SIMTRA available at www.draft.ugent.be/

[17] Macias-Montero M, Garcia-Garcia F J, Alvarez R, Gil-Rostra J, Gonzalez J C, Cotrino J, Gonzalez-Elipe A R and Palmero A 2012 J. Appl. Phys. 111054312

[18] van Hattum E D, Palmero A, Arnoldbik W M, Rudolph H and Habraken F H P M 2007 Appl. Phys. Lett. 91171501

[19] Thornton J A 1974 J. Vac. Sci. Technol. 11666

[20] Thornton J A 1975 J. Vac. Sci. Technol. 12830

[21] IUPAC, McNaught A D and Wilkinson A 1997 IUPAC Compendium of Chemical Terminology: the Gold Book 2nd edn (Oxford: Blackwell)

[22] Palmero A, Rudolph H and Habraken F H P M 2007 J. Appl. Phys. 101083307

[23] Gonzalez-Garcia L, Barranco A, Muñoz Paez A, Gonzalez-Elipe A R, Garcia-Gutierrez M C, Hernandez J J, Rueda D R, Ezquerra T A and Babonneau D 2010 ChemPhysChem 112205

[24] Müller-Buschbaum P 2009 Applications of Synchrotron Light to Scattering and Diffraction in Materials and Life Sciences (Springer Lecture Notes Physics vol 776) (Berlin: Springer) pp 61-89

[25] González-García L, Parra-Barranco J, Sánchez-Valencia J R, Barranco A, Borrás A, González-Elipe A R, García-Gutiérrez M C, Hernández J J, Rueda D R and Ezquerra T A 2012 Nanotechnology 23205701

[26] Lucas S and Moskovkin P 2010 Thin Solids Films 5185355

[27] www.fundp.ac.be/sciences/physique/pmr/telechargement/ logiciels/nascam

[28] Pötting K, Schmickler W and Jacob T 2010 ChemPhysChem 111395

[29] Álvarez R, Palmero A, Prieto-López L O, Yubero F, Cotrino J, de la Cruz W, Rudolph H, Habraken F H P M and Gonzalez-Elipe A R 2010 J. Appl. Phys. 107054311 